\title{
El placer y la búsqueda del conocimiento entre algunos de los traductores latinos del siglo XII
}

\author{
The pleasure and the search for knowledge in \\ some of the 12th C Western Latin translators
}

Pedro Mantas-EsPaÑA

Universidad de Córdoba

Recibido: 09-04-2013

Aceptado: 29-10-2013

\section{Resumen}

A lo largo del siglo XII, un grupo de intelectuales del Occidente latino se afanan por conocer los nuevos textos de filosofía y ciencia traducidos al árabe. Asistimos a un proceso de trasmisión del saber que se concreta en una búsqueda y un deseo firme por acceder al contenido y las novedades que podían aportar estos textos. No se trata sólo de un proceso de ampliación del saber sino de un cambio de perspectiva en los intereses del conocimiento. Una nueva visión que se manifiesta a través de un cambio de actitud intelectual - una búsqueda "racional" de las causas que permitan explicar el orden de la naturaleza - y un vívido interés por leer algunos textos científicos y filosóficos que se interpretan como un saber repleto de nuevas respuestas. En este sentido, hago referencia a Adelardo de Bath, Hermann de Carintia y Domingo Gundisalvo, cuya labor consideramos paradigmática de esa búsqueda y deseo de saber "racional", que permite delinear con más precisión el fenómeno de la transmisión del saber, y el placer que se deriva de éste.

Palabras clave: transmisión del conocimiento, búsqueda del saber, traductores s. XII, Adelardo de Bath, Hermann de Carintia, Domingo Gundisalvo.

\begin{abstract}
Throughout the twelfth century, a group of Western Latin intellectuals are struggling to learn the new philosophy and science texts translated into Arabic. We are witnesses of a process of transmission of knowledge that represent a search and a strong desire in the access to the contents and innovations that those texts could provide. It is not just a process of knowledge increasing but a change of perspective concerning the interests of knowledge.
\end{abstract}


A new perspective which illustrates a change of intellectual attitude - a 'rational' searching for causes which tries to explain the order of nature - and a vivid interest in approaching to some scientific and philosophical texts that they see as a new knowledge filled with new answers. In this sense, I make several references to Adelard of Bath, Hermann of Carinthia and Dominicus Gundissalinus, whose work we consider paradigmatic of a search and desire for 'rational' knowledge, that enables us to delineate more precisely the phenomenon of transmission of knowledge, and the pleasure they find within it.

Keywords: Transmission of Knowledge, Searching for Wisdom, 12th C. Translators, Adelard of Bath, Hermann of Carinthia, Dominicus Gundissalinus.

\section{Presentación}

En la tradición clásica, la felicidad como beatitudo, a diferencia de otras formas de placer, siempre se consideró emancipada de cualquier sentido perturbador, reconociéndose no sólo como el más alto de todos los placeres, sino también como el objetivo real de una vida humana al encuentro con la felicidad.

En las últimas décadas hemos asistido a una recuperación del concepto felicitá mentale que se puede constatar en aportaciones como las de Pierre Hadot ${ }^{1}$ y Maria Corti. ${ }^{2}$ El placer del conocimiento aparece hoy como un campo de investigación relevante y prometedor en la historia de la filosofía medieval, como así lo atestiguan algunas publicaciones clave de los últimos veinte años; entre ellas, baste citar los ensayos de Aertsen, Speer y Emery, ${ }^{3}$ Luca Bianchi, ${ }^{4}$ Alain de Libera, ${ }^{5}$ Juliusz Domanski ${ }^{6}$ y Ruedi Imbach, ${ }^{7}$ así como las aportaciones presentadas por Bettetini y Paparella en el Congreso Le felicità nel Medioevo, ${ }^{8}$ junto con algunos de los últimos trabajos de Gianfranco Fioravanti. ${ }^{9}$ Investigaciones que, a pesar de

\footnotetext{
${ }^{1}$ Hadot, P. , Ejercicios espirituales y filosofía antigua, Madrid: Siruela, 2006.

2 Corti, R., La felicità mentale: nouve prospettive per Cavalcanti e Dante, Giulio Einaudi, Torino 1983. Para este ensayo, véase también Scritti su Cavalcanti e Dante: la felicità mentale, percorsi dell'invenzione, e altri saggi, Torino: Giulio Einaudi, 2004.

3 Aertsen, J. A., Emery, K. y A. Speer, Nach Der Verurteilung Von 1277: Philosophies Und Theologie an Der Universitat Von Paris Im Letzen Viertel DES 13. Jahrhunderts, Berlin: Walter De Gruyter, 2000.

4 Bianchi, L., Il vescovo e i filosofi. La condanna parigina del 1277 e l'evoluzione dell'aristotelismo scolastico, Bergamo: Lubrina, 1990.

5 de Libera, A., Penser au Moyen Âge, Seuil, Paris, 1991; Metaphysique et noétique. Albert le Grand, Paris: Vrin, 2005.

6 Domanski, J., La philosophie, théorie ou manière de vivre? les controverses de l'Antiquité à la Renaissance, Paris: Les éditions du Cerf, 1996.

7 Imbach, R., Dante, la philosophie et les laïcs. Initiations à la philosophie médiévale, Paris: ed. du Cerf, 1996.

8 Bettetini, M. y Paparella F.D., (eds.), La felicità nel Medioevo, Louvain-la-Neuve: FIDEM Textes et Études du Moyen Âge, 31, 2005.

9 Fioravanti, G., "La felicità intellettuale, storiografia e precisazioni”" en M. Bettetini y F.D. Paparella (eds.), op. cit., 1-12.
} 
sus diferencias específicas, comparten no sólo un notable conocimiento de las fuentes sino el empeño por reconstruir una historia del pensamiento medieval como expresión de las constelaciones históricas que lo determinan y hacia donde se proyecta.

La reflexión sobre el placer que aporta el conocimiento forma parte indiscutible de uno de los pilares esenciales del pensamiento clásico; en el pensamiento medieval, con toda su gama de modulaciones, esta tradición subsiste aunque es a partir de un período que podemos hacer coincidir con la primera mitad del s. XII, cuando se va a ir desarrollando un fenómeno intelectual especialmente interesante donde la felicidad se matiza como fruto del placer intelectual que produce la búsqueda del conocimiento.

Como muy bien hizo notar Dimitri Gutas ${ }^{10}$, hablar del movimiento de las traducciones del árabe al latín en el s. XII es tratar de un movimiento histórico muy complejo donde se pueden distinguir $-\mathrm{y}$ en esto Gutas asume la división de Richard Lemay 11 - tres etapas en el desarrollo de las traducciones en la Península Ibérica: el período inicial, donde destaca Adelardo de Bath y Pedro Alfonso; el período que comienza con el final de la segunda década del doce y se prolonga hasta la aparición en Toledo de Gerardo de Cremona, pero donde las figuras de Hermann de Carintia y Robert de Ketton son clave; y por último, el período que representa el trabajo realizado por Domingo Gundisalvo y los maestros con los que despliega su actividad en Toledo.

Pero, independientemente de la necesaria revisión y puntualización que esta división requiere, pues son muchos los trabajos que sobre este fenómeno se están publicado en los últimos años, ${ }^{12}$ en este trabajo he mantenido la estructura de los tres grupos. No obstante, no creo aventurado afirmar que en todos ellos se vislumbra un común denominador: la relación entre la búsqueda del conocimiento y el placer que esta exploración le aporta a quien la practica. No obstante, en la labor desempeñada por estos intelectuales es necesario tener en cuenta una gradación que se manifiesta en distintos aspectos: por un lado, el aumento progresivo de complejidad temática; por otro lado, el acceso a fuentes cada vez más sugerentes y ricas tanto por su fidelidad con respecto al original como por el enriquecimiento de las fuentes que las transmiten; finalmente y de manera esencial, por la complejidad intelectual o filosófica desde la que se aborda la búsqueda del saber y los trabajos de traducción. En este último aspecto y en el contexto del fenómeno de las traducciones en la Península Ibérica a lo largo del s. XII, la progresión alcanzará uno de sus puntos culminantes con la obra de Domingo Gundisalvo.

\footnotetext{
10 Gutas, D., "What was there in Arabic for the Latins to Receive? Remarks on the Modalities of the Twelfth-Century Translation Movement in Spain", en Wissen über Grenzen: Arabisches Wissen und lateinisches Mittelalter, A. Speer y L. Wegener (eds.), Berlin: Walter de Gruyter, 2006, 3-4.

11 Cfr. Lemay, R., "Dans l'Espagne du XIIe siècle. Les traductions de l'arabe au latin”, Annales. Economies, Sociétés, Civilisations 18 (1963), 639-665.

12 Trabajos entre los que destacan especialmente los ensayos dedicados por Charles Burnett a este ámbito de la investigación - algunos de los más recientes son citados a lo largo del artículo - u obras de conjunto tan relevantes como la ya citada edición de Speer y Wegener.
} 


\section{En torno a la curiosidad y el deseo intelectual}

El contenido al que responde el título de este trabajo encierra un problema de interpretación sobre el que se ha reflexionado muy recientemente, y en el que creo de especial interés poder detenernos. Como introducción al tema que da título a mi trabajo comenzaré con una breve referencia en torno al documento que Thomas Ricklin y Marc-Aeilko Aris escribieron como marco teórico y presentación del "estado de la cuestión" en el reciente XIII Congreso de la Sociedad Internacional para el Estudio de la Filosofía Medieval, ${ }^{13}$ pues supone un ejercicio de reflexión particularmente interesante sobre algunos de los principios que están presentes en parte de la actividad del pensamiento medieval; aspectos que casi siempre han quedado relegados e incluso olvidados, aunque fueron y son tan relevantes como algunos de los grandes tópicos abordados en la historia del pensamiento medieval. En este sentido, no he podido evitar reflexionar en torno a estos preliminares y la hermenéutica desde la que ambos autores justificaban la organización del citado Congreso, especialmente en el contexto intelectual desde el que planteo mi trabajo, donde considero necesario delimitar el sentido y la relación de la idea de búsqueda como placer, dentro de un análisis sobre la exploración del conocimiento centrado en la primera mitad del siglo XII.

En el contexto del documento de Ricklin y Aris al que hacía referencia, se rememora aquel pasaje del Filebo en el que Sócrates defiende que «los placeres de los conocimientos no implican mezcla con dolores y de ningún modo corresponden a la mayoría de los hombres sino a un número muy exiguo."14 Porque, efectivamente, para Platón la pura felicidad de los filósofos es un bien muy escaso y difícil de alcanzar, como así lo corrobora el fragmento de las Tusculanas donde Cicerón afirma que la mente, en tanto se conoce a sí misma "[...] se siente unida a la mente divina, por lo que se colma de un gozo insaciable."15 Ambas citas pretenden aludir a la reflexión sobre las virtudes que se desprende o que se sitúa en el origen del conocimiento, una disquisición que trata de aportar luz en el dilema sobre la búsqueda, el descubrimiento y la conquista del saber unidos al sentido de la felicidad.

Pero, mientras que en el pensamiento clásico y, decíamos, también en el pensamiento medieval, esta vinculación confirma y promueve la fructífera posibilidad de un vínculo entre conocimiento y felicidad, esta relación no se manifiesta mediante un proceso que, partiendo del deseo de conocer, perciba su búsqueda como una fuente de placer. Porque, a diferencia del sentido que posee la beatitudo, y en la relación con el conocimiento, la intervención del placer y el deseo suele tratarse como un caso especial y con problemáticas connotaciones en la búsqueda de un sumo bien - tal vez porque confirmar un papel tolerable para

\footnotetext{
13 Ricklin T. y Aris M., SIEPM 2012 Freising, "Pleasures of Knowledge", International Congress of Medieval Philosophy. August 20-25, 2012. Las actas y el citado documento introductorio aparecerán publicadas a lo largo del año 2014.

14 Platón, Filebo, 52b, trad. Ma Ángeles Durán, Madrid: Gredos, 1992, 96. Uno de los estudios más recientes donde se aborda el concepto filosófico del placer en el pensamiento griego clásico y su relevancia para la reflexión contemporánea sobre dicho concepto lo encontramos en D. Wolfsdorf, Pleasure in Ancient Greek Philosophy. Key themes in ancient philosophy, Cambridge, New York: Cambridge University Press, 2013.

15 Cicerón, Discputaciones tuscalanas, V, trad. A. Medina, Madrid: Gredos, 2005, 429.
} 
el placer y el deseo requiere no tanto de una delimitación del placer, cuanto de un concreción sobre la clase de volición que implicamos en nuestros deseos.

En un pasaje La ciudad de Dios, Agustín de Hipona insiste en que:

[...] ningún otro impulso excita al hombre a filosofar, sino el deseo de ser bienaventurado, y lo que únicamente hace bienaventurado es sólo el fin del bien; luego ninguna otra causa hay para filosofar sino el fin del sumo bien; por lo cual, la secta que no sigue algún fin del bien, no debe llamarse secta filosófica. ${ }^{16}$

En este mismo sentido, Boecio insistirá en el problema de la relación entre el deseo del bien, y un modo de interpretar el placer que sólo puede justificarse cuando es resultado de la consecución del bien, cuando el deseo del bien convierte el placer en felicidad. No es extraño, pues, que partiendo de una imprecisa inspiración aristotélica, algunos de los grandes maestros medievales relacionen el bien con la felicidad que se deriva de una vida dedicada a la actividad intelectual y al placer que aporta un reconocimiento discursivo de la realidad. En cualquier caso, junto a estas perspectivas nos hemos detenido en una cuestión mucho menos tratada y que se deriva del análisis que aquí abordamos, a saber, la praxis intelectual. Es en este sentido que nos preguntamos: ¿qué podemos decir acerca de un placer que provenga o se derive de la praxis intelectual?

La cuestión me parece pertinente por varias razones, me detendré sólo en una: un recorrido desde la artes del trivium hacia el quadrivium, o un proceso que, partiendo de un buen adiestramiento en la retórica y la dialéctica se eleve hasta la especulación en torno a la física y la contemplación matemática y metafísica, sigue excesivamente anclado en el programa intelectual planteado por Aristóteles (Metafísica, VI, I) - un programa al que justamente tratarán de responder o matizar algunas de las obras de eruditos y filósofos de la primera mitad del s. XII a los que nos referimos en este trabajo. Al mismo tiempo, no podemos obviar el sentido instrumental de la praxis, que tanta relevancia va a adquirir en el proceso de redescubrimiento de la naturaleza y de las técnicas necesarias para la observación - como es el caso del cálculo, el diagnóstico y pronóstico que tiene lugar en la investigación astronómica y médica desde los siglos once al trece. En este contexto, la actividad traductora será, en muchas ocasiones, fuente de placer intelectual en sí mismo - piénsese en el reto que podría suponer la consecución de un fin instrumental equiparable al proceso mismo de su realización, ya sea en el trabajo con el cómputo, la observación astronómica o los tratamientos médicos y farmacológicos.

Si la felicidad es la felicidad de la práctica filosófica, una actividad que sitúa al filósofo entre Dios y los hombres (Marsilio Ficino); si partiendo de una tradición clásica que se extiende a lo largo de toda la Edad Media, el conocimiento ha podido concebirse y proyectarse como placer y beatitud, creemos especialmente interesante poder investigar cómo algunos filósofos y eruditos profesionales integrarán esta concepción, cómo la respaldan, agudizan, idealizan o la ocultan.

Los modelos estructurales que definen la adquisición del conocimiento pueden considerarse como vías a través de las cuales se organiza el proceso de adquisición del conoci-

16 Agustín De Hipona, La ciudad de Dios, XIX, 1, trad. S. Santamaría y M. Fuertes, Madrid: BAC, 2007, p. 824. 
miento. En este sentido, suele distingue entre, por un lado, el conocimiento generado a través de la tradición y la recepción; por otro, la producción del conocimiento como la reconstrucción de una estructura inteligible que pertenece a la realidad como un todo; finalmente, el conocimiento como una representación de la realidad en su conjunto que puede derivar en una nueva forma de conocimiento.

Si en lugar de tratar los modelos estructurales de adquisición del conocimiento, establecemos una diferenciación centrada en los modos de conocimiento, es entonces cuando hablamos de la contemplatio como un modo de conocimiento que, entre otras particularidades, denota una relación entre la producción del conocimiento y su vinculación afectiva; un modo de concebirla donde pueden diferenciarse distintas áreas de investigación - desde el mundo viviente hasta la realidad material como un todo - o donde el conocimiento se concreta como resultado de la investigación en torno a la conexión originalmente determinada de la estructura de la realidad, persiguiendo identificar sus relaciones y causas. Esto último forma parte de la interpretación que doy al contenido de este artículo, aunque, en realidad, nuestra investigación se centra en la perspectiva de aquellos intelectuales para quienes el deseo no es sino la manifestación de un impulso que los anima a perseguir las causas que explicarían aquello que se desea conocer, y una investigación que aspira a alcanzar la satisfacción por medio del saber.

Las técnicas que se desarrollan en la Edad Media para apoyar el trabajo de los eruditos pero, sobre todo, las aspiraciones que éstas generan, quedan muy bien reflejadas en la intención y las expectativas que se originan a través del trabajo de las traducción. No obstante, he dado más importancia al impulso y al proceso de producción del conocimiento desde una relación entre intellectus, desiderium y curiositas, porque el ámbito desde el que planteo mi trabajo se centra en la percepción que estos intelectuales dedicados a la transmisión y producción de nuevos saberes extraen sobre su trabajo, que perciben como parte de un indudable desarrollo del intelecto humano y de su racionalidad.

En el ámbito del s. XII, intellectus y curiositas no pueden desligarse de la consumada producción de conocimiento y del gozo que produce el desarrollo de dicho conocimiento en el sentido de una perfectio. Pero, si no es fácil poder concretar el sentido preciso de la vinculación entre la curiositas e intellectus en relación con la búsqueda y el placer del conocimiento, tal vez resulte mucho más difícil poder imaginar qué actitud y sensaciones produce entre algunos de los estudiosos que abordamos en este trabajo. Por suerte para nosotros, contamos con algunos testimonios tan claros como manifiestos.

\section{Adelardo de Bath y su búsqueda del saber}

Comencemos con Adelardo de Bath, el representante más destacado dentro del primer período de eruditos y traductores del s. XII al que anteriormente aludíamos pues, en realidad, el segundo grupo al que Gutas hace referencia (Robert de Ketton y Hermann de Carintia, entre ellos) no pueden explicarse contextual y adecuadamente sin antes tratar sobre Adelardo y la influencia que su trabajo ejerció en este contexto.

A estas alturas no es poco lo que conocemos sobre la actividad de Adelardo como erudito, traductor y autor original. En realidad, la actitud ante el conocimiento que se despren- 
de de la mayor parte de su vida y de la relación entre su biografía intelectual y los resultados que surgieron de ésta, tal vez sean el mejor ejemplo que puede citarse con respecto a la primera mitad del siglo doce. Me limitaré tan sólo a citar algunos de los aspectos que caracterizan su búsqueda del conocimiento, una búsqueda que es, al mismo tiempo, un magnífico ejemplo en el desarrollo de las transferencias del conocimiento en la Edad Media. ${ }^{17}$

Podría decirse que la obra más conocida de Adelardo, las Quaestiones Naturales, ${ }^{18}$ no es sólo una primera y extensa evidencia de un giro de los tratados sobre la naturaleza hacia la física sublunar, sino también, una magnífica representación de algunas de las características que le son pertinentes a la mayoría de los textos relacionados con la fase del desarrollo de la filosofía de la naturaleza que se desarrolla en la primera mitad el s. XII: de un lado, el género de cuestiones que se muestran y la integración o el uso de nuevas fuentes, cuya procedencia hay que situar en Sicilia y el sur de Italia, la costa Norteafricana y Oriente Medio; de otro lado, el origen textual de obras o traducciones árabes a partir de textos clásicos. Pues aunque es cierto que ya en el s. VIII, en el ámbito de la cultura latina existían ciertas colecciones de fuentes textuales, lo importante ahora es el nuevo interés con el que son leídas y, cómo no, el modo con el que es acogido el nuevo material documental. En cualquier caso, la diferencia más significativa hay que buscarla en la naturaleza de las cuestiones que se formulan y en las respuestas o soluciones que se dan a las preguntas. Pues tratan de explicar los fenómenos naturales, el porqué, las causas que les son inherentes o en las que se basan. La búsqueda del conocimiento en Adelardo se puede resumir aludiendo a las fases más importantes del proceso de adquisición de sus conocimientos: la primera transcurre entre sus años de formación en Tours y como tutor en Laon, en esta etapa la formación de Adelardo se centra en lo que él mismo denomina los gallica studia, p.e., cuando Adelardo increpa a su sobrino, que se encontraba formándose en Laon: "Lo que ignoras en los estudios galos se te hará accesible tras los Alpes."19

Se trata de una etapa que podemos identificar con su interés por el estudio de las artes del trivium (aunque fue en Tours donde se produjo su primera inmersión en el estudio de la astronomía), a este período pertenece una de sus obras originales titulada Sobre lo idéntico y lo diferente. Desde Laon, Adelardo viaja durante siete años persiguiendo y formándose en los estudios de los árabes. De nuevo, Adelardo se dirige a su sobrino y le pregunta a su sobrino:

Recuerdas, querido sobrino, que hace siete años, cuando me despedía de ti (todavía eras casi un niño) junto a mis otros alumnos en los estudios galos en Laon, acordamos entre nosotros que yo

17 En relación con este aspecto y en el contexto del trabajo intelectual realizado por Adelardo en su investigación en torno a la naturaleza, véase Mantas-España, P., "La naturaleza en Adelardo de Bath", en J. Merinhos y M. Lázaro (eds.) Pensar a natureza. Problemas e respostas na Filosofia Medieval (séculos IX-XIV), Porto: Faculdade de Letras da Universidade do Porto, 2011, 27-45.

18 Adelardo de Bath, Quaestiones Naturales (QN), en Adelard of Bath, Conversations with his Nephew: on the same and the different, questions on natural science, and on birds, ed. y trad. Ch. Burnett, cols. I. Ronca, P. Mantas-España, B. van den Abeele, Cambridge: Cambridge University Press, 1998, 81-235.

19 Adelardo De Bath, Sobre lo idéntico y lo diferente, trad. P. Mantas-España, Revista Española de Filosofia Medieval 5 (1998), 249-64 y 7 (2000), 163-79. 
investigaría en los estudios de los árabes según mi capacidad, y que tú alcanzarías una no menor destreza en la inseguridad de las afirmaciones de los galos. ${ }^{20}$

O en este otro celebrado pasaje donde la búsqueda del conocimiento, de las causas que pueden explicar la realidad desde la razón, se oponen a un exclusivo recurso al principio de autoridad que él detecta en las enseñanzas tradicionales, y que no se percibe en su encuentro intelectual con los estudios de los árabes. De nuevo, Adelardo increpa a su sobrino diciéndole:

Pues, con la razón como guía, algo he aprendido de mis maestros árabes, mientras que tú algo muy distinto, pues te dejas guiar por un cabestro, cautivado por los ornamentos con que la autoridad se engalana. Pues, ¿de qué otro modo se puede denominar la autoridad sino como cabestro? [... la autoridad] por si sola, no puede obtener credibilidad para un filósofo, ni puede ser aducida para este propósito. ${ }^{21}$

Desde su vuelta a Inglaterra (antes de 1135) y hasta el final de su vida, Adelardo va a ir completando su labor como introductor y traductor de obras científicas, especialmente de obras matemáticas; al mismo tiempo, irá ejerciendo una notable influencia entre jóvenes intelectuales de Inglaterra o Francia - a través de sus contactos con la casa de Platagenet o debido a la recepción de sus escritos en Chartres - lo que va a favorecer un clima de interés por la lectura y la traducción de los textos árabes de la Península Ibérica, Sicilia y Oriente Medio.

Cito uno de los pasajes de su obra sobre el ábaco donde, de nuevo, propone un paso más acelerado desde los estudios del trivium hacia el quadrivium. En esta ocasión, de un modo muy original:

Adelardo, el más insignificante acólito de los filósofos, saluda a su H. ¿Recuerdas aquella ocasión cuando nos encontramos compartiendo la 'mesa' filosófica? Algunos manjares se hallaban ante nosotros, pero nos encontrábamos sentados a la derecha, aparte de los otros huéspedes. Nuestros comensales más próximos devoraban la segunda parte comiendo tres platos al mismo tiempo, mientras yo trataba de introducir entre tus labios unos bocados de un plato dividido en cuatro partes. Como ponías objeciones a todas las viandas que yo intentaba que comieses, al advertir que otros huéspedes se las habían dejado y tú no las habias probado antes, bebiste primero un antídoto pitagórico [esto es, el ábaco o mensa Pythagorea] 22

\title{
4. Algunos rasgos característicos de la búsqueda y el placer del conocimiento. La actividad de los eruditos, traductores y filósofos en la Península Ibérica
}

Con este enunciado hago referencia a la citada segunda y tercera fase en la actividad de los traductores en la Península Ibérica, aludiendo no tanto ni fundamentalmente a los tra-

\author{
20 QN, 90. \\ 21 Ibid., 102. \\ 22 Adelardo De Bath, Regule abaci, en Ch. Burnett, Adelard of Bath: An English Scientist and Arabist \\ of the Early Twelfth Century, London: Warburg Institute Surveys and Texts 14, 1987, 35.
}


ductores sin más, sino a algunos conocidos y celebrados intelectuales que, en algunos casos, podemos calificar como auténticos eruditos y filósofos.

Ya en la primera mitad del s. XII, la versión árabe del Gran Tratado de Claudio Ptolomeo, el reverenciado Almagesto, se había convertido en uno de los objetivos intelectualmente más deseados entre algunos de los personajes vinculados directa o indirectamente con la historia de la equívocamente denominada "escuela de traductores de Toledo". Entre el grupo de traductores que llegan a la Península con la intención de acceder a distintos textos clásicos dignos de la más absoluta veneración se encontraban, entre otros, maestros, eruditos o filósofos como Robert de Ketton, Herman de Carintia, Gerardo de Cremona o Domingo Gundisalvo. De las distintas versiones en árabe del Almagesto, en la Europa del s. XII se tenía acceso a dos manuscritos: el de al-Ḥajjāj b. Yūsuf b. Mațar y el de Ishāa b. Hunayn, realizados a principios y finales del s. IX respectivamente - éste último, no obstante, en la versión de principios del s. X realizada por Thābit ibn Qurra.

Como muy bien recordaba Charles Burnett en una ponencia sobre la tradición árabe y latina del Almagesto 23 - rememorando la clásica y magistral obra de Charles Haskins sobre la historia de la ciencia medieval 24 - si Ketton, Carintia y Cremona acometieron la traducción del texto a partir del árabe, y es la traducción de Gerardo de Cremona (anterior a 1175) la que se convertiría en la más popular, fue un anónimo y apasionado traductor siciliano quien a mediados del doce acometía la traducción a partir de una versión en griego. En un pasaje introductorio, el autor declaraba:

[...], cuando estaba sudando con la medicina [con los estudios de medicina] en Salerno escuché que el rey de Sicilia habia enviado a Constantinopla a uno de sus emisarios llamado Aristipo 25 - que habia recibido el Almagesto en señal de regalo del emperador y lo había traído a Palermo. Enardecido por la esperanza de poseer algo que durante mucho tiempo tanto había deseado, no estremecido por los rugidos de Sicilia, atravesé Caribidis [el estrecho de Mesina], y bordeé los cauces ardientes del Etna, buscando aquél de quien esperaba la consecución de mi deseo. 26

23 Burnett, Ch., “The Arabic and Latin Tradition of Ptolemy's Almagest”, conferencia impartida en Tokio el 9 de Diciembre de 2009 en el Seminario The Transmission of Scientific Knowledge: Greek, Syriac, Arabic, and Latin organizado por el "Proyecto MEXT: Joint Research Center for Islamic Area Studies" desarrollado por The Waseda Organization of Islamic Area Studies de la Universidad Waseda de Tokio, en colaboración con la Universidad Sangyo de Kyoto.

${ }^{24}$ Haskins, Ch. H., Studies in the History of Mediaeval Science, Cambridge (MA), Harvard University Press, 1927, 121.

25 De Enrico Aristippo hoy sabemos con alguna certeza que fue archidiácono de Catania en torno a 1155; que en la primavera del 1156 se encontraba con el ejército de rey Guillermo en Benevento donde, ese mismo año, comenzó la traducción del Fedón de Platón. Entre 1158 y 60 formó parte de la embajada que Guillermo envió a Constantinopla; en respuesta a esta embajada y en señal de reconocimiento, el emperador Manuel I Comneno envió al rey normando una serie de códices griegos entre los que se encontraba el Almagesto. Sobre la relación entre Guillermo I y Aristippo véase H. Takayama, The Administration of the Norman Kingdom of Sicily, Leiden: Brill, 1993, 95-114. Véase también A. M ${ }^{\mathrm{a}}$ Ieraci Bio, "La redazione greca inedita d'una 'questio' salernitana", en V. BoudonMillot, A. Guardasole y C. Magdelaine (eds.), La science médicale antique: nouveaux regards, Paris: Beauchesne, 2008, 253.

26 "[...] cum Salerni medicine insudassem audiens quendam ex nunciis regis Sicilie quos ipse Constantinopolim miserat agnomine Aristipum largitione susceptos imperatoria Panormum 
En este mismo contexto, Burnett también cita el texto donde Robert de Ketton se dirige a Hermann de Carintia y le recuerda el compromiso mutuo por acometer una traducción del Almagesto - curiosamente, también aquí se conjuga una forma verbal de insudo en alusión a las dificultades de la traducción:

Aunque después de Euclides, habría sudado más a gusto en la "Cosmometría"27 de Teodosio y un libro sobre las proporciones, para posibilitar una aproximación más conveniente al "Almagesto", que es el objetivo principal de nuestro estudio, y para no silenciar nuestra amistad a causa de mi pereza, no obstante $[\ldots]^{28}$

El texto continúa con un elogio de Robert a su amigo Hermann y la confirmación de haber terminado la traducción de la obra que éste le había recomendado: los Iudicia de alKindi.

En ambos pasajes no sólo se exhibe un extraordinario deseo por acometer la lectura del Almagesto, también se pone de manifiesto una aspiración intelectual por un saber que da la impresión de quedar muy lejos del fin último que inicialmente pudo justificar la labor desarrollada por algunos traductores. Veamos.

Aunque al principio del proceso de búsqueda y traducción del saber clásico y helenístico, los textos más perseguidos no fueron las obras de los grandes filósofos o científicos de la antigüedad, sino obras de astronomía y astrología, tratados que versaban sobre la naturaleza de las cosas, y donde no se sabía diferenciar adecuadamente entre ciencia y ocultismo; no obstante, parte de esta actividad inicial surgía de un interés de carácter eminentemente religioso, como ocurre, por ejemplo, cuando en 1141 Pedro el Venerable mantiene un encuentro con Robert de Ketton y Hermann de Carintia en el valle del Ebro, un emplazamiento donde el abad de Cluny les pide que traduzcan algunos textos musulmanes. Entre las obras que traducirán, y en respuesta a la petición de Pedro el Venerable, se encuentra el Corán. Se trata, por tanto, de un interés que muchos casos posee un carácter dual: el deseo de traducir los textos escritos, transcritos o interpretados por los árabes servirán, por un lado, para un mejor conocimiento de la religión musulmana y de aquellos que la profesan pero, al mismo tiempo, para acceder a un vastísimo material textual que contribuya a ensanchar los límites intelectuales, y despejar un horizonte filosófico y científico que entonces comienza a percibirse como claramente insuficiente. Este interés dual se observa, por ejemplo, en las traducciones elaboradas por Gundisalvo y sus colaboradores en Toledo.

Como Alexander Fidora ha apuntado, ${ }^{29}$ un ejemplo muy clarificador es el que se extrae del prólogo dedicatoria en la traducción del libro VI de los Libri naturales de Avicena (el

transvexisse, rei diu multumque desiderate spe succensus, Scilleos latratus non exhorrui, Caribdim permeavi, ignea Ethne fluenta circuivi, eum queritans a quo mei finem sperabam desiderii.". Cfr. Haskins, op. cit., 191-193.

27 Con respecto a la Cosmometria, se han sugerido dos posibles obras, el De Sphaeris y el De habitatibilibus.

28 "Quamquam post Euclidem Theodosii Cosmometrie libroque proportionum libencius insudarem, unde commodior ad Almaiesti, quo precipuum nostrum aspirat studium, pateret accessus, tamen ne per meam segniciem nostra surdesceret amicicia, [...]."

29 Fidora, A., Domingo Gundisalvo y la teoría de la ciencia arábigo-aristotélica, Pamplona: EUNSA, 2009, 235-238. 
tratado De anima) traducido en colaboración con Avendauth (Abraham ibn Dāwūd): la racionalización, i.e., un esfuerzo por hacer comprensible los contenidos de la fe, buscando argumentos que fortalezcan la fe, un interés de carácter religioso, junto a una actividad intelectual netamente movida por el deseo de saber. En este último caso y de modo distinto a como se percibe en Pedro el Venerable, podemos descubrir la idea de una búsqueda del conocimiento orientado no tanto por fines religiosos, una actitud que, en realidad, podemos retrotraer más de un siglo. Pues, qué podríamos decir de una personalidad tan prominente como Gerberto de Aurillac, quien mucho antes que Pedro el Venerable ya se había interesado e involucrado en esta búsqueda del saber de la que estamos tratando.

A su conocimiento de las fuentes árabes sobre astronomía y cómputo contribuyó su estancia en la Península Ibérica (donde hay que destacar sus vínculos con el obispado de Vic). Se sabe que el interés hacia estas cuestiones se inicia en la época en que Juan de Gorze fue nombrado embajador de la corte de Otto I en Córdoba (954), allí pudo haber reunido algunos manuscritos a los que Gerberto habría tenido acceso. En este sentido, podemos considerarlo un precursor del deseo por conocer la ciencia que se producía o se transmitía en árabe dentro de la Península Ibérica, pero sobre todo, porque su actividad científica no parece justificarse como parte de una función doctrinal. También en este sentido, nos interesa especialmente el precedente que Gerberto representa, pues su actividad científica posee características muy similares a las que ponen de manifiesto algunos de los miembros más reconocidos entre los grandes traductores del doce. Pudiendo presentarse como uno de los precursores del renovado interés hacia la ciencia, no guiado en su praxis por motivos religiosos o doctrinales, aunque dicha praxis se concrete por medio de una actividad de colaboración con problemas prácticos de carácter religioso.

¿Qué animaba a Gerberto a buscar e investigar en las fuentes del saber científico matemático, una actitud similar a la que compartían nuestros traductores y eruditos?

Hasta donde podemos afirmarlo, Gerberto supone un paso previo pero no muy distante, ni en el tiempo ni su actividad, de los eruditos a los que Nadja German ${ }^{30}$ sitúa - usando una expresión empleada por Burnett - en el período de la física antes de la Física. ${ }^{31}$ Un período en el que integramos a Abón de Fleury junto al propio Gerberto, Hermann de Reichenau, el Pseudo-Columbano o Garlando el Computista, en el contexto de una astronomía solar y lunar (el computo), donde tanto Garlando como Hermann distinguen claramente entre la autoridad antigua o eclesiástica, y la naturaleza o la razón - estos últimos no dudarán en actuar según la naturaleza, renovando reglas y cálculos de poca precisión y desarrollando algunas novedades.

Si aceptamos que la idea de beatitudo, a la que aludimos al principio, integra un sentido que supone la búsqueda de bienes y fines últimos, entonces y en relación al intellectus,

\footnotetext{
${ }^{30}$ Algunas de las conclusiones de sus investigaciones sobre cómputo y astronomía fueron presentadas en el IV.e Congrès Européen d'Études Médiévales "Coexistence et Coopération au Moyen Âge", celebrado en Palermo en Junio 2009, para un trabajo más elaborado véase Germann, N., "Natural Philosophy in Earlier Latin Thought", en R. Pasnau (ed.), The Cambridge History of Medieval Philosophy, vol. I, Cambridge: Cambridge University Press, 2009, 219-231.

31 Para el contexto y sentido de esta expresión, $c f r$. Burnett, Ch., "Physics before the Physics: Early Translations from Arabic of Texts Concerning Nature in Mss British Library, Additional 22719 and Cotton Galba E IV", Medioevo, 27 (2002) 53- 109; y N. Germann, op. cit., pp. 219-220.
} 
desiderium y curiositas, la fuerza que impulsa a estos eruditos hacia el deseo de conocimientos desde una actitud nueva habría que concretarla advirtiendo que, en estos casos o al menos en una de sus vertientes intelectuales, los fines "últimos" serían de carácter eminentemente intelectual y práctico. Por tanto y retomando lo ya apuntado en la introducción, si vamos tratando de aclarar qué persiguen, qué mueve a estos eruditos y filósofos ${ }^{32}$ del doce podemos aventurar lo siguiente:

$\mathrm{Su}$ esfuerzo por comprender la naturaleza tal vez se mueva entre el deseo por formar parte de aquello que se quiere conocer, tal vez entrar en relación intelectual con el Creador de la naturaleza, e incluso alcanzar a comprender a su Creador. Pero, ¿es alguna de estas vertientes relacionales del saber la que produce felicidad, se trata de una felicidad que se percibe como placer? Parece que si existe un sumo bien al que aspirar en el proceso del conocimiento, este sumo bien es, en parte, el conocimiento por sí y en sí mismo aunque, insisto, sólo en parte. Veamos.

Cuando más arriba he aludido a la praxis como un medio o un primer nivel en el proceso de ascenso al conocimiento especulativo, aún no había señalado algo que en este contexto me parece esencial: la distinción hoy clásica y asumida en la historiografía filosófica entre filosofías especulativas de la Naturaleza y ciencia ha contribuido a una explicación sobre la formación y las características definitorias de la ciencia moderna; en el caso que abordamos, hemos de reconocer que cuando nos enfrentamos al trabajo de algunos de estos traductores filósofos es necesario replantear algunos de nuestros conceptos y la hermenéutica desde la que los formulamos. Es claro que la realización práctica forma parte consustancial del trabajo de traducción, pero también lo es el modo como determinadas aplicaciones tanto geométricas como mecánicas, químicas o farmacológicas que se crean, se desarrollan o se perfeccionan a partir de los tratados traducidos y de las interpretaciones que generan estas traducciones, se manifiestan como algo más que un camino o un adiestramiento previo hacia la especulación matemática y metafísica. El programa real que se desprende de la labor de algunos de estos traductores nos habla de una praxis especulativamente

\footnotetext{
32 Efectivamente, hablo de filósofos, pues aunque en el doce los puestos de honor entre los grandes pensadores latinos que reflexionan sobre la naturaleza tradicionalmente han quedado reservados para los maestros de la Escuela de Chartres, no hay que olvidar que también algunos de sus discípulos y algunos de quienes mantuvieron una estrecha relación con Chartres desarrollaron una actividad filosófica incuestionable: este fue el caso de, entre otros, Hermann de Carintia, alumno de Thierry de Chartres, y cuyo De essentiis nadie duda a estas alturas en clasificar como una obra ineludible. Véase Hermann de Carintia, De essentiis, ed. Ch. Burnett, Leiden - Köln: Brill, 1982. Con respecto a Domingo Gundisalvo baste decir que obras como De immortalitate animae, el Tractatus de anima o el De divisione philosophiae serán de una influencia notable no sólo por sí mismas sino, en el caso de esta última, por la repercusión que tendría para la configuración del plan de estudios de la recién fundada Facultad de Artes de la Universidad de París. Para ediciones recientes de dos de estas obras, véase El Tractatus de Anima atribuido a Dominicus Gundissalinus, ed. y trad. C. Soto del Real y $\mathrm{M}^{\mathrm{a}} \mathrm{J}$. Soto Bruna, Pamplona: EUNSA, 2009; Domingo Gundisalvo, Über die Einteilung der Philosophie / De divisione philosophiae, ed. y trad. A. Fidora y D. Werner, Freiburg: Herder, 2007, 40-49. Del tratado De immortalitate animae contamos con la traducción al castellano de N. Kinoshita incorporada en su estudio sobre Gundisalvo a partir de la edición clásica de G. Bülow (BGPhMa, II, 3), véase N. Kinoshita, El pensamiento filosófico de Domingo Gundisalvo, Salamanca: Universidad Pontificia de Salamanca, 1988, 129-149.
} 
guiada, y de unos resultados que son, por sí mismos, especulación científica y a la vez, praxis cientifica. Por supuesto, no hablamos de una praxis científica en sentido moderno, obviamente no, aunque sí como anticipaciones de rasgos de modernidad; unos precedentes que nos llevan a enlazar el s. XII con la línea que marca el punto de transición entre los siglos XIV y XV. En este sentido me parece muy ilustrativo el trabajo de Fidora que he citado, no sólo porque demuestra que, frente a las tesis de Grignaschi, 33 Gundisalvo cita (De divisione philosophiae) la Física recurriendo al Aristoteles arabus ${ }^{34}$ y no a la tradición greco-latina - un Aristóteles que él mismo habría traducido directamente del árabe, pues ni Gerardo de Cremona ni Miguel Scoto habían traducido aún esta obra. Pero, sobre todo, porque permite constatar algo que se repite constantemente en el proceso de las traducciones como transmisión e intercambio del saber: las modificaciones de conceptos y los filtros intermedios producen un extraordinario enriquecimiento sobre las fuentes originales. El Aristóteles que Gundisalvo lee no es, por supuesto, un Aristóteles “puro" sino un Aristóteles neoplatonizado por Avicena; pero al hacer esta lectura, Gundisalvo desarrolla unas variantes al texto de Aristóteles (Física, II, 7, 198a) que, en este caso concreto al que alude Fidora, despliegan un orden alternativo, precisamente, al problema de la distinción entre los "quehaceres", los pragmateia, de la investigación científica del que, como apuntábamos al principio, ya había tratado Aristóteles en su Metafísica (VI 1, 1026 y XII 1, 1069a - 1069b).

Conviene recordar que lo que intentamos aclarar responde a las distintas preguntas ya planteadas, pero fundamentalmente a estas tres cuestiones: ¿es la búsqueda del conocimiento una búsqueda del placer?, ¿de qué placer hablamos?, ¿qué papel ocupa el deseo y la curiosidad en todo este proceso? En su magistral trabajo sobre Gundisalvo, Fidora no duda en afirmar que:

No parece admisible explicar la recepción explícita de la epistemología de Aristóteles por la mera curiosidad intelectual de algunos eruditos situados en la periferia del mundo latino, y mucho menos como consecuencia de la simple disponibilidad de nuevas fuentes [...]. Antes bien, la recepción de Aristóteles en el terreno de la teoría del conocimiento y de las ciencias resulta del desarrollo consecuente de problemas teóricos ya largamente discutidos en la propia tradición, $[\ldots]$ cuyo potencial para ofrecer nuevas soluciones se hallaba agotado por completo $[\ldots] .35$

Finalmente, si al tratar del placer del conocimiento, en tanto que actividad intelectual en la que el deseo y la curiosidad impulsan una buena parte de su labor, nos detenemos en trabajos tan recientes como la edición que Mews y Crossley han dedicado a las comunidades y redes de aprendizaje en la formación intelectual de la identidad europea entre los

33 Grignaschi, M., "Le De divisione philosophiae de Dominicus Gundissalinus et les Quaestiones IIV in Sextum Metaphysicorum de Jean de Jandun", en S. Knuuttila, R. Tyorinoja y S. Ebbesen (eds.), Knowledge and the Sciences in Medieval Philosophy. Proceedings of the Eighth International Congress of Medieval Philosophy, Helsinki: Yliopistopaino, 1990, pp. 53-61.

34 Fidora, A., "Notas sobre Domingo Gundisalvo y el Aristoteles Arabus", Al-Qantara XXIII, 1 (2002) 201-208.

35 Fidora, A., Domingo Gundisalvo y la teoría de la ciencia arábigo-aristotélica, op. cit., 232-233. 
siglos doce y dieciséis y, más en concreto, en algunos de los ensayos centrados en el s. XII, 36 su lectura ratifica la idea de una apertura a los nuevos o renovados saberes que aportan los textos traducidos. Una apertura que no parece se encuentre sometida a algún tipo de autocensura de carácter religioso que pudiese limitar la actividad de los traductores. Por el contrario, la atmósfera en la que se desenvolvía esta actividad en el Toledo de la segunda mitad del doce - descrita como una atmósfera vibrante ${ }^{37}$ - no supone un tipo de tolerancia de carácter político, sino la tolerancia intelectual que uno encuentra en los ejemplos ya citados. En cualquier caso, un diálogo mediado por la filosofía que, resultado de traducciones filosóficas y científicas como las producidas en el Toledo de Domingo Gundisalvo, Abraham Ibn Daūd o Gerardo de Cremona, en el valle del Ebro o distintas latitudes de al-Andalus, ponen de manifiesto un deseo expreso por penetrar en el descubrimiento de nuevos saberes y reestructurar algunos de los ya conocidos.

En esta búsqueda del conocimiento que parte del deseo por desvelar nuevas respuestas, la curiosidad es elemento clave en el impulso de un deseo que deriva en placer intelectual. Una indagación que parte de una sospecha o de una constatación: que en la investigación en torno a la naturaleza y las cuestiones filosóficas esenciales, un programa de estudio o un plan de trabajo que se base en una tradición textual como la que representa Boecio - por poner un buen ejemplo - se percibía como algo ya agotado. Se necesitaba, pues, de un redescubrimiento de los textos clásicos y las nuevas aportaciones árabes, vía el trabajo de traducción e interpretación. Ello supondría un salto cuantitativo pero, sobre todo, cualitativo que contribuirá a desentrañar más razonablemente el orden admirable de la realidad que se aspiraba a conocer. En este contexto, el siglo doce y la actividad de los traductores e intérpretes de ciertas obras transmitidas, comentadas o reinterpretadas por los árabes, representa el comienzo de un cambio paradigmático en la actividad intelectual que, en sus desarrollos posteriores y siglos más tarde, contribuirá a la generación de la ciencia y el pensamiento moderno.

Al abordar el concepto de placer vinculado a la curiosidad y el deseo - en relación con una felicidad que se matiza como fruto del placer derivado de la búsqueda del conocimiento - nos hemos detenido en una actitud intelectual que se produce en el contexto del fenómeno de las traducciones del s. XII en la Península Ibérica, y en cómo sus protagonistas manifiestan los rasgos de dicha vinculación: hemos advertido que, por un lado, la curiosidad y el deseo incrementan el atractivo de una fértil relación entre praxis y especulación; al mismo tiempo, hemos ilustrado cómo de esta actitud intelectual también se desprende un uso crítico del conocimiento capaz de someter a revisión e incluso enfrentarse al principio de autoridad.

\section{Pedro Mantas-España}

Universidad de Córdoba

fs1maesp@uco.es

\footnotetext{
36 Mews C. J. y Crossley, J. N., Communities of Learning: Networks and the Shaping of Intellectual Identity in Europe, 1100 - 1500, Turnhout: Brepols, 2011. Para el contexto que estamos tratando, véase especialmente Ch. Burnett, "Communities of Learning in Twelfth-Century Toledo" (9-18) y A. Fidora, "Religious Diversity and the Philosophical Translations of Twelfth-Century Toledo" (19-36).

37 Ibid. 18
} 